\title{
Reducing blood culture contamination rate: a quality assurance project in a Malaysian tertiary hospital
}

\author{
Siti Roszilawati Ramli ${ }^{1,2}$, Sabarina Zahari², Azura Sadri' ${ }^{2}$, Zahrotul Farihah Aziz², Alex Francis ${ }^{2}$ \\ 1. Institute for Medical Research, Malaysia \\ 2. Hospital Raja Permaisuri Bainun, Ipoh, Malaysia
}

doi: 10.3396/IJIC.v10i2.016.14

\begin{abstract}
Blood cultures contamination is a common scenario in Malaysian hospitals. Reducing the contamination rate of blood culture will reduce false-positive blood culture, prevent inappropriate antibiotic prescription and reduce patient management cost. This is a 6 months study specifically focused on the effects of changing the type of skin antiseptics used ( $70 \%$ isopropyl alcohol plus $2 \%$ chlorhexidine replacing $70 \%$ isopropyl alcohol plus povidone-iodine) and improving the knowledge and techniques of blood culture taking in a tertiary hospital. The remedial measures introduced were successful in achieving $4.34 \%$ contamination rate, which is $2.03 \%$ less than pre-remedial contamination rate $(6.37 \%)$. There was a statistically significant reduction in the number of contaminants $(p=0.02)$ with five-fold rise in the significant blood cultures results. Better choice of skin antiseptics and good blood culture knowledge and technique may contribute to reduce number of contamination rate in blood culture.
\end{abstract}

Keywords: Blood-borne pathogens; Blood specimen collection; Quality assurance

\section{Background}

Blood cultures are the most important laboratory tests performed in the diagnosis of serious infections, and would lead to a definitive diagnosis and therapy against the causative organism. Ambiguous results and contamination among blood cultures has become apparent, costly, and confusing the clinicians. Despite the limitations, blood culture remains the 'gold standard' for the detection of bacteremia. Reducing the contamination rate of blood culture will reduce false-positive blood culture, prevent inappropriate antibiotic prescription and reduce laboratory and patient management cost.

Blood culture is a microbiology test done when a patient suspected to have bacteremia. Blood is drawn from patient in an aseptic procedure using skin disinfectant/s, one or more times and is tested in a laboratory to identify any microorganism present in the blood. Contaminants are considered if only

\section{Corresponding author}

Siti Roszilawati Ramli

Institute for Medical Research, Malaysia

Email: srosz77@gmail.com 
one positive blood culture grow coagulase negative Staphylococcus (CoNS), aerobic and anaerobic diphtheroids, Micrococcus spp., and Bacillus spp.

Contamination of blood cultures is considered common in certain centres, representing up to half of all positive blood cultures. ${ }^{1}$ Another study reported that the actual blood contamination rate ranged between 0.6- $6 \%{ }^{2}$ As far as the authors are concerned, to date there is no published rate on blood culture contamination rate in Malaysian hospital. This study was carried out to determine the contamination rate of blood cultures in our centre and reduce the rate by improving the technique of disinfection and type of skin antiseptic used for blood culture preparation.

\section{Methods}

We conducted a prospective, nonrandomised interventional study at Hospital Raja Permaisuri Bainun, Ipoh, Perak. This institution has 990 inpatient beds and an emergency department. It provides tertiary care for a community with a population of approximately 650,000 in a central area of Ipoh. From $1^{\text {st }}$ October 2011 till 31 $1^{\text {st }}$ March 2012 all patients in medical wards who were over 13 years of age and who were suspected of having bacteraemia were enrolled in this study.

During a 6-month period all blood cultures ordered by attending physicians in our internal medicine ward were obtained by the house officers according to the following protocol. At least two sets of blood samples were recommended to be collected from patients who had symptoms or signs of bacteraemia requiring blood cultures taken from them. We excluded oncology patients, renal failure patients and patients with devices such as central venous catheters, chemoports and dialysis catheters.

Blood samples were drawn from antecubital veins using 21-gauge needles and disposable syringes. From each patient, one sample was collected (including both aerobic and anaerobic bottles) and all were handled and processed in an identical manner except for venepuncture site preparation. All blood cultures included in this study were obtained from inpatients who were clinically suspected to have sepsis or for monitoring response to antibiotics.
During the first three months, blood culture was obtained after strict antiseptic cleansing, recommended by most authors. This consisted of application of $70 \%$ alcohol for one minute followed by application of povidone-iodine solution ( $1 \%$ available iodine) for an additional minute using a sterile applicator in a concentric manner, then venepuncture was performed without palpating the vein again. During the second phase of the study, blood culture was obtained after application of $70 \%$ alcohol for one minute followed by application of $2 \%$ chlorhexidine in $70 \%$ alcohol for few seconds using a sterile applicator. Venepuncture was performed after the solution on the skin was dried.

In between the two phases of the study, an intervention of education programs and blood culture best practice campaign were carried out for mostly all housemen from medical wards. The education programs implemented included lectures and demonstrations of correct blood culture techniques with pre and post written tests to evaluate their knowledge and understanding. Besides that, other media such as posters, reminder cards and reminder chits on blood culture bottles were incorporated into the campaign to keep the housemen aware of the good practice in blood culture taking (Figure 1).

In each venepuncture 8 to $10 \mathrm{ml}$ of blood were recommended to be inoculated into each blood culture bottle (BD Bactec Plus aerobic/F and BD Bactec Plus+ Anaerobic/F, Becton Dickinson and Company, Sparks, MD 21152), after replacing the needle and swabbing the rubber stoppers of blood culture bottles with $70 \%$ alcohol. All blood cultures underwent standard incubation and processing in the Microbiology Unit in Department of Pathology, using a BACTEC 460 radiometric analyser (Johnson Laboratories, Towson, Maryland) for 5 days, and declared negative if no growth was detected by then. A blood culture was considered positive if an organism was isolated from a single or both media. The definition of false positive results was based on clinical judgement made from multiple factors including clinical data such as patient's history, physical findings; clinical course laboratory analysis and type of organism/s isolated, culture results from other body sites and percentage of positive culture results. ${ }^{3-4}$ 
1. Substitution of $2 \%$ Chlorhexidine in $70 \%$ as skin disinfectant for blood cultures in medical wards.

2. Lectures and demonstration on best practices in collecting blood cultures.

3. Posters placed in medical wards showing optimum procedures of blood cultures including skin disinfection.

4. Handing out reminder cards (showing summarised optimum procedure in blood collection) to housemen

5. Reminder chit on optimum procedures of blood cultures pasted on blood culture bottle

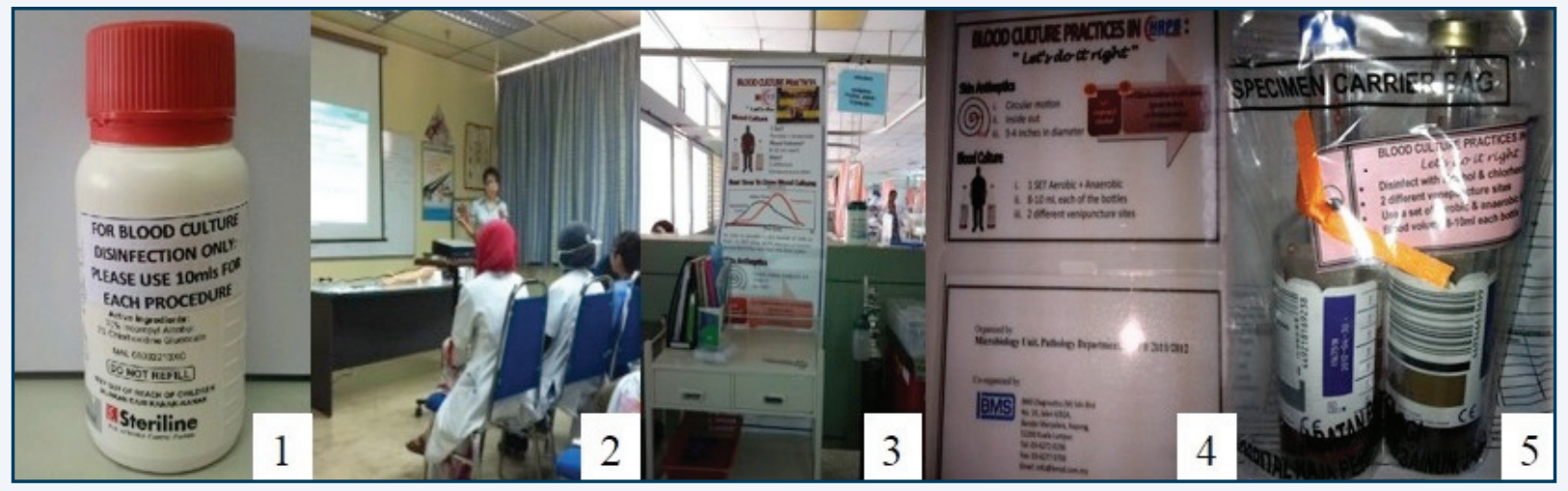

Figure 1. Implementation of Remedial Measures

Statistical analysis included comparing proportions by the Chi Square test. P-value of less than 0.05 was considered statistically significant.

\section{Results}

Result of this study demonstrated that the Medical Department (the main contributor of blood culture in the centre) has reduced its contamination rate by $2.03 \%$, (from $6.37 \%$ pre-intervention to $4.34 \%$ postintervention). The difference is significant with a $p$-value of 0.02 . There is $49 \%$ reduction in percentage of contaminant (Table I). The post intervention rate has successfully passed beyond the targeted standard contamination rate of $5 \%$. In this study, we also noted that there is five-fold rise in the number of significant blood cultures at post remedial. This could be due to the increase of volume and number of blood cultures sent for a patient, which were included in the subjects of improvement in the remedial measures, as the campaign endorsed for a comprehensive improvement in the practice of blood culture. The parameters of volume and number of cultures were not recorded in our study as the intended improvement was measured as a whole through the contamination rate and true positive culture rate.

\section{Discussion}

Blood culture contamination is a renowned issue in most hospitals in Malaysia, however little is known on the seriousness and how much measures can be done to tackle the problem. In this study, we found that there was a significant reduction in contamination rate of blood culture when there is substitution of $2 \%$ chlorhexidine in $70 \%$ alcohol for skin disinfection. This

Table I. Comparison on pre and post remedial measures

\begin{tabular}{lrr}
\multicolumn{1}{c}{ Blood Cultures } & Pre-Remedial Measures & Post-Remedial Measures \\
\cline { 2 - 3 } & (Oct-Dec 2011) & (Jan-Mar 2012) \\
\hline Total Blood Cultures & 1380 & 1266 \\
\hline Total Positive & 114 & 195 \\
\hline Total True Positive Blood Cultures & 26 & 140 \\
\hline Total Contaminated Blood Cultures & $88(6.37 \%$ of total $)$ & $55(4.34 \%$ of total) \\
\hline & $(77.2 \%$ of positive cultures $)$ & $(28.2 \%$ of positive cultures) \\
\hline
\end{tabular}


result was anticipated as most studies have proven on its practicality due to its property that dries quickly and effectiveness in lowering the contamination rate..$^{5-6}$

Besides differentiating the effect of two types of skin antiseptic, this study aimed to ensure a good practice in blood culture taking, by a comprehensive approaches to improved understanding, knowledge and awareness on blood culture technique. Thus, we had decided that by providing educational input in the remedial measures the associated factors in lowering the contamination rates can be strengthened. In fact, best practice guidance issued by the Department of Health, London, ${ }^{7}$ states that blood cultures should only be collected by members of staff who have been trained in the procedure and whose competence in blood culture collection has been assessed. Housemen involved in this campaign had undergone compulsory practical training by the Infection Control Unit and undergone 1 hour tutorial comprised of lectures and demonstration and all had passed post tutorial test. The inclusion of simple measures such as enclosing advisory leaflets, posters and providing reminder chit on blood culture bottles could be effective strategies. ${ }^{8}$

Throughout the study, the main challenge was keeping in tract of new housemen to be included in the program as fast turnaround time of housemen rotation in medical department as well as flexi-hours working hours were the greatest challenge. Somehow we achieved 95\% coverage of housemen from medical department for the campaign. This was achieved by personal phone calls and call letters. A serial lectures and demonstrations were conducted and they showed very good understanding and knowledge based on their post questionnaires scores.

Based on the result of this study, we conclude that better choice of skin disinfectant may contribute to reduce number of contamination rate in blood culture. Thus, we highly recommend the usage of $2 \%$ chlorhexidine in $70 \%$ alcohol for skin disinfection especially in the critical wards such as intensive care unit and high dependency unit. Continuous education and training as well as adherence to guideline on appropriate blood culture techniques may enhance knowledge, skill and awareness among healthcare workers.
There are few other suggestions that could be considered for future strategies for good blood culture practice as well as for reduction of blood culture contamination rates which includes using a 'closed' system in which blood is drawn directly into vacuum culture bottles, ${ }^{9}$ employing the service of phlebotomy teams, ${ }^{10}$ providing direct feedback of contamination rates to those who perform blood cultures, ${ }^{11}$ as well as monitoring blood culture rates of respective departments in a regular meeting of Hospital Infection Control Committee.

\section{Conclusion}

Blood cultures are still an important diagnostic tool for septic workout, nevertheless blood culture contamination remains a chronic and recalcitrant problem. There are many aspects of blood culture taking that one's should delve into for improvement of true positive culture. A multidisciplinary approach with various strategies concerning on awareness, continuous education, technique improvement as well as contamination rate monitoring may bring a significant long term solution.

\section{Acknowledgements}

The authors thanked Director General of Health Malaysia for the permission to publish this work. We also would like to thank Dato' Dr Norain Karim, Dato' Dr K Chandran, Infection Control Unit, Staff of Medical Department, as well as staff of Microbiology Unit for the aspiration and cooperation in making this QAP project a success. We also would like to thank BMS Marketing and Steriline Sdn Bhd for their support.

\section{References}

1. Bates DW, Goldman, L, Lee TH. Contaminant Blood Cultures and Resource Utilization. The Consequences of False Positive Results. JAMA 1991; 3: 365-369. http://dx.doi.org/10.1001/ jama.1991.03460030071031

2. Hall KK and Lyman JA. Updated Review of Blood Culture Contamination. Clin. Microbiol.Rev 2006; 19: 788-802. http:// dx.doi.org/10.1128/CMR.00062-05

3. MacGregor RR, Beaty HN. Evaluation of positive blood cultures: guidelines for early differentiation of contaminated from valid positive cultures. Arch Intern Med 1972; 130: 84-87. http://dx.doi.org/10.1001/archinte.1972.03650010072013

4. Weinstein MP, Reller LB, Murphy JR, Lichtenstein KA. The clinical significance of positive blood cultures: a comprehensive analysis of 500 episodes of bacteremia and fungemia in adults. I. Laboratory and epidemiologic observations. Rev Infect Dis 1983; 5: 35-53. http://dx.doi. org/10.1093/clinids/5.1.35 
5. Mimoz O, Karim A, Mercat A, et al. Chlorhexidine compared with Povidone-lodine as skin preparation before blood culture a randomized controlled trial. Ann of Intern Med 1999; 131: 834-838. http://dx.doi.org/10.7326/0003-4819-131-11199912070-00006

6. Madeo M, Barlow G. Reducing blood-culture contamination rates by the use of a $2 \%$ chlorhexidine solution applicator in acute admission units. Journal of Hospital Infection 2008; 69(3): 309. http://dx.doi.org/10.1016/j.jhin.2008.03.009

7. Department of Health Saving Lives: taking blood cultures a summary of best practice. Department of Health: London 2007.
8. Madeo M, Jackson T, Williams C. Simple measures to reduce the rate of contamination of blood cultures in accident and emergency. Emerg Med Journal 2005; 22: 810-811. http:// dx.doi.org/10.1136/emj.2005.003079

9. Ellner PD. System for inoculation of blood in the laboratory. Appl Microbiol 1968; 16: 1892-1894.

10. Eskira S, Gilad J, Shlaeffer P, et al. Reduction of blood culture contamination rate by an educational intervention. Clin Microb and Inf 2006; 12: 818-821.

11. Gibb AP, Hill B, Chorel B, Brant R. Reduction in blood culture contamination rate by feedback to phlebotomists. Archives of Pathology and Lab Med 1997; 121: 503-507. 